\title{
Interoperabilidade Social e Colaboração: um Mapeamento Sistemático da Literatura
}

\author{
Rian das Dores Alves ${ }^{1}$, Rita S. P. Maciel ${ }^{2}$, José Maria N. David ${ }^{1}$ \\ ${ }^{1}$ Departamento de Ciência da Computação \\ Universidade Federal de Juiz de Fora (UFJF) - Juiz de Fora, MG - Brazil \\ ${ }^{2}$ Departamento de Sistemas e Computação \\ Universidade Federal da Bahia (UFBA) - Salvador, BA - Brazil \\ rian.alves@ice.ufjf.br, rita.suzana@ufba.br, jose.david@ufjf.edu.br
}

\begin{abstract}
Collaborative systems have a high degree of complexity due to the requirements they must meet. Among them, we can mention the support for social interoperability, which needs social contextualization of users to support interoperability in collaborative systems. As a result, we aimed to enrich the context of activities and increase the users' awareness and understanding of the performed activities through these systems. However, Social Interoperability is still little explored and needs to be analyzed. Thus, this work aims to carry out a systematic mapping study to analyze Social Interoperability issues in developing of collaborative systems.
\end{abstract}

Resumo. Sistemas colaborativos apresentam um elevado grau de complexidade devido aos requisitos que devem atender. Entre eles, podemos mencionar o suporte à interoperabilidade social, que utiliza da contextualização social dos usuários a fim de apoiar a interoperabilidade em sistemas colaborativos. Como resultado, é possível enriquecer o contexto das atividades e aumentar a percepção e compreensão dos usuários em relação às atividades realizadas por meio desses sistemas. Contudo, a Interoperabilidade Social ainda é pouco explorada e precisa ser analisada. Assim, o objetivo é realizar um mapeamento sistemático para analisar aspectos da utilização da Interoperabilidade Social no desenvolvimento de sistemas colaborativos.

\section{Introdução}

São altos o consumo e o compartilhamento de dados, informações e procedimentos em muitos contextos da sociedade [Chen and Quan-Haase 2020]. Diversos serviços utilizam as tecnologias de informação para agilizar processos e facilitar a vida dos atores envolvidos. Em processos de colaboração, os sistemas utilizados, são considerados cada vez mais complexos, pois devem suportar indivíduos com percepções diferentes, interagindo de diversas maneiras em contextos distintos [Neiva et al. 2016] [David and Maciel 2009]. Como exemplo, podemos citar os sistemas que apoiam as cidades inteligentes e a indústria 4.0. Assim, os sistemas colaborativos precisam estar alinhados aos recursos e serviços que interoperam, de modo a compreender os dados e oferecer suporte às atividades cotidianas da sociedade. Sendo assim, é necessário que sejam construídas soluções de interoperabilidade, eficientes e eficazes, que apoiem as interações entre sistemas colaborativos e os stakeholders envolvidos.

A interoperabilidade pode ser considerada como a capacidade de compartilhar 
dados entre diferentes sistemas, dispositivos, plataformas e ecossistemas [Neiva et al. 2015] [Soares 2010]. Dado que a complexidade de interoperar aumenta à medida que dados de diferentes fontes e contextos são explorados, a interoperabilidade não pode ser vista apenas como dispositivos que se conectam a fim de enviar e receber dados [Kasunic 2001]. Sistemas colaborativos podem requerer diferentes níveis de interoperabilidade para alcançar a interoperabilidade plena [Maciel et al. 2017]. Os níveis de interoperabilidade sintática, semântica e pragmática podem não ser suficientes para suprir às necessidades envolvidas no processo de colaboração. Principalmente em relação às interações humano-computador-humano, que são centrais no ambiente colaborativo [Neiva et al. 2016].

As interações sociais dos indivíduos por meio de sistemas de comunicação e informação podem ser importantes fontes de conhecimento [Olshannikova 2017]. Da mesma maneira, o conhecimento oriundo dessas interações pode apoiar a contextualização de informações e atividades em um processo de interoperabilidade. Logo, a partir das interações sociais apoiadas por diferentes fontes de dados dos indivíduos em um ambiente colaborativo, este trabalho propõe a Interoperabilidade Social como um nível de interoperabilidade a ser tratado. A proposição acerca da Interoperabilidade Social é devido à possibilidade de uma contextualização social de processos e interações dos diferentes atores e tecnologias utilizadas nesses processos. Essa contextualização pode apoiar a compreensão dos dados trocados e também a automatização de processos para a interoperabilidade de diferentes sistemas e serviços envolvidos nas atividades de colaboração.

A falta de discussão sobre Interoperabilidade Social ocasiona uma não unanimidade em sua definição e poucas soluções são discutidas na literatura. Entretanto, as abordagens encontradas consideram a interação do ser humano com os dados, assim como o contexto social do indivíduo, pontos principais para a interoperabilidade social [Ruban et al. 2019] [Li et al. 2013]. Garlapati e Biswas (2012), por exemplo, definem a interoperabilidade social como a capacidade de compreensão do compartilhamento de dados técnicos e da consistência semântica de forma a apoiar ao fluxo de trabalho na área de cuidados em saúde. Contudo, a ausência de um consenso no entendimento desse tipo de interoperabilidade constitui uma barreira para proposição de soluções. Esse entendimento entre os participantes dos sistemas colaborativos é importante para o provimento de soluções de interoperabilidade.

Apesar de existirem trabalhos que abordem a Interoperabilidade Social [Ruban et al. 2019] [Li et al. 2013], não foram identificados estudos secundários que explorem o estado da arte desse tipo de interoperabilidade. Portanto, devido à ausência de estudos acerca da Interoperabilidade Social, e possíveis aplicações desse nível de interoperabilidade entre sistemas colaborativos, um Mapeamento Sistemático da Literatura (MSL) foi conduzido. Foram elaboradas questões de pesquisa para identificar publicações sobre Interoperabilidade Social, os domínios de aplicações tratados, soluções desenvolvidas e aplicação dessas soluções em processos de colaboração.

Assim, o artigo está organizado da seguinte forma: a Seção 2 apresenta conceitos fundamentais sobre o tema; a Seção 3 apresenta o processo do mapeamento; a Seção 4 explora as questões exploradas; a Seção 5 faz uma análise da interoperabilidade social em sistemas colaborativos e a Seção 6 conclui o trabalho. 


\section{Interoperabilidade Social}

Nesta seção é realizado o embasamento teórico acerca de Interoperabilidade Social, cujo objetivo é apoiar o entendimento desse tipo de interoperabilidade. A HL $7^{1}$ (Health Level Seven) define a Interoperabilidade de Processos e a Interoperabilidade Social como sinônimos. Entretanto, os fatores sociais são apenas uma parte dos requisitos de processos interoperáveis que são tratados em um domínio de saúde. Sadeghi et al. (2012) enfatizam que sistemas de saúde interoperáveis precisam considerar, além das tecnologias e processos, os atores envolvidos. Os autores reconhecem a interoperabilidade social como requisito fundamental para que ocorra a Interoperabilidade de Processos e a colaboração efetiva em uma equipe voltada para a assistência médica. Neste contexto, a interoperabilidade social está focada na troca de informações entre pacientes, e entre pacientes e especialistas por meio de uma rede social em um ambiente de colaboração em saúde. Para que ela seja devidamente apoiada, devem ser considerados dados referentes ao contexto e às particularidades de cada ator envolvido no processo, pois ela foca no trabalho humano, nas relações das pessoas e seus ambientes [Garlapati and Biswas 2012].

Landsbergen Jr and Wolken Jr (2001) abordam a Interoperabilidade Social sob o ponto de vista das questões de dimensões política, econômica e organizacional, além da técnica. Segundo os autores, todas essas questões quando envolvidas no compartilhamento de informações definem a Interoperabilidade Social. Assim, para compreender melhor esse tipo de interoperabilidade, é interessante caminhar por essas dimensões a fim de conhecer os dados interoperados e os tipos de interoperabilidade que são empregados nos sistemas, organizações e redes privadas ou públicas que envolvem essas dimensões.

Ruban et al. (2019) definem interoperabilidade social a partir de um modelo de interoperabilidade orientado a problemas para trocar informações entre sistemas de $e$ learning. O modelo contém um número de níveis, sendo a Interoperabilidade Social um desses níveis, que é garantido pela adoção de normas e regras de comportamento uniformes na sociedade e no ambiente educacional. Li et al. (2013) também apresentam a Interoperabilidade Social como um nível de interoperabilidade. Os autores propõem níveis de interoperabilidade baseados na semiótica organizacional, sendo a Interoperabilidade Social o nível que alinha os aspectos sociais, como cultura, normas, meio ambiente e padrões de comportamento dos atores, para solucionar quaisquer conflitos de coesão. Além disso, permite que a intenção ou propósito do remetente leve a uma consequência para o destinatário, que pode ser um compromisso social, obrigação ou norma (interoperabilidade pragmática).

A partir do embasamento teórico desta seção, pode-se observar que não há um consenso acerca da Interoperabilidade Social. De maneira que esse tipo de interoperabilidade se apresenta como um nível que compreende os aspectos sociais no processo de interoperabilidade, ou como uma perspectiva social sobre os dados, ou simplesmente, como a troca de dados de cunho social por meio de tecnologias de informação. Sendo assim, em busca do estado da arte acerca de Interoperabilidade Social, com definições e soluções para esse tipo de interoperabilidade, um mapeamento sistemático foi conduzido.

\footnotetext{
${ }^{1}$ Wiki HL7 Group (Acessado em abril de 2020).
} 


\section{Mapeamento Sistemático da Literatura}

A estratégia adotada para encontrar e identificar estudos e soluções relevantes no que se refere a Interoperabilidade Social foi a Busca Híbrida [Mourão et al. 2017]. A estratégia e abordagem foram adotadas devido a necessidade de encontrar trabalhos que apresentem soluções relevantes para a Interoperabilidade Social, ainda não explorada em diferentes domínios. Com esse tipo de revisão busca-se aumentar a quantidade e qualidade dos trabalhos encontrados, a partir do Snowballing, dos artigos selecionados da biblioteca digital Scopus ${ }^{2}$. Essa biblioteca foi escolhida devido à abrangência de bibliotecas por ela indexadas, às ferramentas de auxílio à leitura de resumos e palavras-chave, além de ser considerada uma das maiores plataformas digitais de publicação científica [Mourão et. al 2017]. A Figura 1 ilustra o processo referente à estratégia de Busca Híbrida adotada neste MSL.

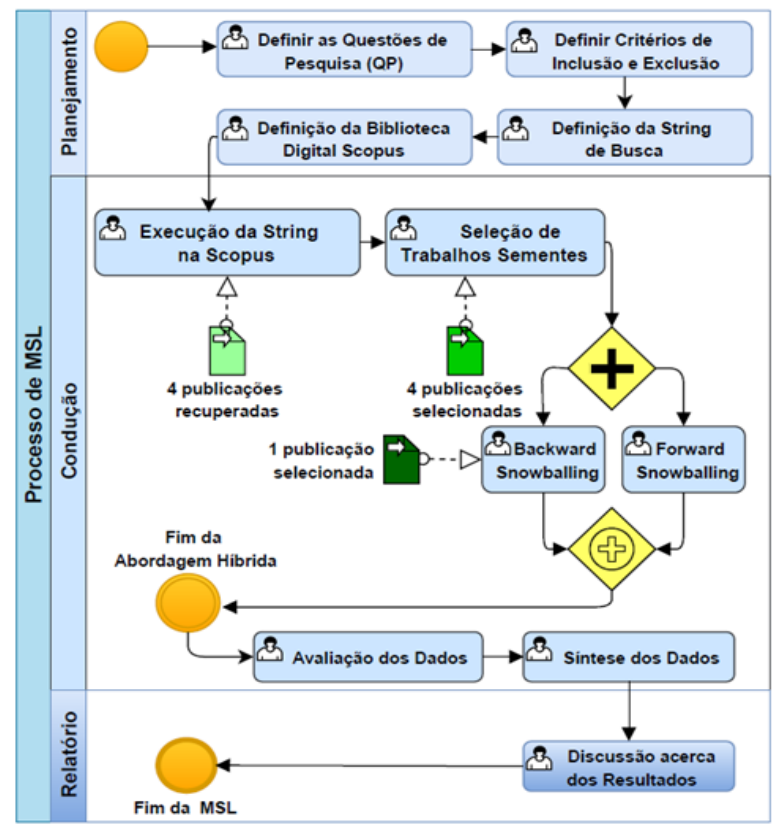

Figura 1. Processo de Mapeamento Sistemático [Mourão et al. 2017]

\subsection{Planejamento}

O objetivo deste MSL é identificar abordagens que apoiem, ou possam servir como base para apoiar a interoperabilidade social em sistemas colaborativos.

\subsubsection{String de Busca}

Para a formalização da string, e consequentemente das questões de pesquisa (QP), utilizase os critérios do método PICOC (Population, Intervention, Comparison, Outcome, Context) sugerido por Petticrew e Roberts (2005). A Tabela 1 dispõe dos elementos do método. Esses elementos contribuem para a geração da string de busca executada na Scopus, e configurada da seguinte maneira:

("social interoperability") OR ("social interoperate") AND ("collaboration" OR “collaborative systems" OR "groupware") OR ("tool” OR "software" OR "service"OR "system"OR "platform"OR "framework" OR "method" OR "model” OR “ontology" OR “approach”)

\footnotetext{
2 Scopus (https://www.elsevier.com/solutions/scopus), accessed April, 2020.
} 
Tabela 1 - PICOC aplicado ao MSL.

\begin{tabular}{|c|c|c|}
\hline PICOC & Termos Definidos \\
\hline Population & $\begin{array}{c}\text { Soluções que abordam } \\
\text { interoperabilidade }\end{array}$ & Interoperability OR Interoperate \\
\hline Intervention & $\begin{array}{c}\text { Soluções que abordam } \\
\text { Interoperabilidade Social }\end{array}$ & Social Interoperability OR Social Interoperate \\
\hline Comparison & Sem comparação & \\
\hline Cutcome & $\begin{array}{c}\text { Soluções (método, técnica, } \\
\text { modelo, ferramenta, plataforma, } \\
\text { estrutura) }\end{array}$ & $\begin{array}{c}\text { Tool OR Software OR Service OR System } \\
\text { OR Platform OR Method OR Model OR } \\
\text { Framework OR Ontology OR Approach }\end{array}$ \\
\hline & $\begin{array}{c}\text { Foco nos aspectos } \\
\text { colaborativos }\end{array}$ & $\begin{array}{c}\text { Collaboration OR Collaborative Systems OR } \\
\text { Groupware }\end{array}$ \\
\hline
\end{tabular}

Os termos referentes a População não foram utilizados de maneira singular e foram concatenados aos de Intervenção. Essa decisão foi tomada devido a particularidade e especialidade dos termos buscados $\mathrm{O}$ contexto foi aplicado à string para possibilitar o retorno de possíveis soluções específicas no campo da colaboração. Esta string foi avaliada por especialistas das áreas de interoperabilidade e sistemas colaborativos. As publicações de Hsu (2013) e Vieira et al. (2016) foram utilizadas como controle.

\subsubsection{Questões de Pesquisa}

A Tabela 2 disponibiliza as questões de pesquisa (QP) que este MSL visa responder. Na primeira coluna tem-se a identificação das QP, na segunda coluna as QP e na última o objetivo de cada uma delas.

Tabela 2 - Questões de Pesquisa do MSL.

\begin{tabular}{|c|l|l|}
\hline ID & \multicolumn{1}{|c|}{ Questões de Pesquisa } & \multicolumn{1}{c|}{ Objetivos } \\
\hline QP1 & $\begin{array}{l}\text { Quais são os domínios de aplicação } \\
\text { tratados entre as soluções que adotam } \\
\text { Interoperabilidade Social? }\end{array}$ & $\begin{array}{l}\text { Elaborada para que se tenha um panorama } \\
\text { de todos os domínios de aplicação } \\
\text { abrangidos entre as soluções } \\
\text { selecionadas. }\end{array}$ \\
\hline QP2 & $\begin{array}{l}\text { Como as soluções abordam a } \\
\text { Interoperabilidade Social? }\end{array}$ & $\begin{array}{l}\text { Explorar as abordagens de } \\
\text { Interoperabilidade Social, e como as } \\
\text { soluções são tratadas: Software, Modelo, } \\
\text { Hardware, Middleware, entre outros. }\end{array}$ \\
\hline QP3 & $\begin{array}{l}\text { Como as soluções de } \\
\text { Interoperabilidade Social encontradas } \\
\text { podem apoiar a complexidade existente } \\
\text { no desenvolvimento de sistemas } \\
\text { colaborativos? }\end{array}$ & $\begin{array}{l}\text { Explorar como as soluções de } \\
\text { interoperabilidade encontradas podem ser } \\
\text { aplicadas em um ambiente de colaboração. } \\
\text { Se não abordam, como as soluções } \\
\text { encontradas poderiam ser relacionadas } \\
\text { com processos ou sistemas de } \\
\text { colaboração. }\end{array}$ \\
\hline
\end{tabular}

\subsubsection{Critérios de Inclusão e Exclusão}

O processo usado para incluir ou excluir uma publicação foi organizado em quatro 
critérios de inclusão (I) e quatro critérios de exclusão (E), apresentados na Tabela 3.

Tabela 3 - Critérios de Inclusão e Exclusão.

\begin{tabular}{|l|l|}
\hline \multicolumn{1}{|c|}{ Inclusão } & \multicolumn{1}{c|}{ Exclusão } \\
\hline $\begin{array}{l}\text { I1: Os trabalhos propuseram uma } \\
\text { solução de interoperabilidade social } \\
\text { (método, técnica, modelo, ferramenta, } \\
\text { estrutura) OU }\end{array}$ & $\begin{array}{l}\text { E1: Os trabalhos não propõem uma } \\
\text { solução de interoperabilidade social } \\
\text { (método, técnica, modelo, ferramenta, } \\
\text { estrutura) OU }\end{array}$ \\
\hline $\begin{array}{l}\text { I2: A solução proposta é aplicada em } \\
\text { software OU sistema OU aplicativo OU } \\
\text { serviço OU infraestrutura E }\end{array}$ & $\begin{array}{l}\text { E2: A solução proposta não é aplicada } \\
\text { a um software OU sistema OU } \\
\text { aplicativo OU serviço OU infraestrutura } \\
\text { E }\end{array}$ \\
\hline $\begin{array}{l}\text { I3: Os trabalhos estão escritos em } \\
\text { inglês E }\end{array}$ & $\begin{array}{l}\text { E3: Os trabalhos não estão descritos } \\
\text { em inglês E }\end{array}$ \\
\hline $\begin{array}{l}\text { I4: Os trabalhos são relatados em } \\
\text { Oficina ou Conferência, Revista ou } \\
\text { Diário ou Relatórios Técnicos revisados } \\
\text { por pares. }\end{array}$ & $\begin{array}{l}\text { E4: Os trabalhos não tratam de } \\
\text { publicações revisados por pares em } \\
\text { eventos ou revistas reconhecidos }\end{array}$ \\
\hline
\end{tabular}

\subsection{Condução do MSL}

Nesta seção apresenta-se os estudos selecionados na primeira etapa da Busca Híbrida e nas buscas que utilizam Backward Snowballing e Forward Snowballing. A busca foi realizada em setembro de 2020 e a biblioteca digital escolhida foi a Scopus. Ao executar a string formulada foram retornadas 4 publicações. Entre elas não havia nenhuma publicação duplicada. Os termos foram encontrados nos títulos, resumos ou palavraschave. Cada um desses elementos foi analisado e todas as publicações foram selecionadas para avaliação do material completo. Após a avaliação, as 4 publicações (100\%) foram aptas a compor o conjunto de trabalhos sementes.

Tabela 4 - Publicações selecionadas no MSL.

\begin{tabular}{|l|c|c|}
\hline \multicolumn{1}{|c|}{ Publicações } & Conjunto & Domínio \\
\hline $\begin{array}{l}\text { Integrating ontology technology with folksonomies for } \\
\text { Personalized social tag recommendation [Hsu 2013] }\end{array}$ & Semente & $\begin{array}{c}\text { Sistemas de } \\
\text { Recomendação }\end{array}$ \\
\hline $\begin{array}{l}\text { Smart solutions for low-income buildings rehabilita- } \\
\text { tion: International researches and experiences [Ar- } \\
\text { bizzani et al. 2015] }\end{array}$ & Semente & $\begin{array}{c}\text { Cidades } \\
\text { Inteligentes }\end{array}$ \\
\hline $\begin{array}{l}\text { Developing imaged KOS with the FRSAD model: A } \\
\text { conceptual methodology [Vieira et al. 2016] }\end{array}$ & Semente & $\begin{array}{c}\text { Bibliotecas } \\
\text { Digitais }\end{array}$ \\
\hline $\begin{array}{l}\text { Universal social network bus: Toward the federation } \\
\text { of heterogeneous online social network services [An- } \\
\text { garita et al. 2019] }\end{array}$ & Semente & Redes Sociais \\
\hline $\begin{array}{l}\text { Leveraging the service bus paradigm for computer- } \\
\text { mediated social communication interoperability [An- } \\
\text { garita et al. 2017] }\end{array}$ & $\begin{array}{l}\text { Backward Snow- } \\
\text { balling [Angarita } \\
\text { et al. 2019] }\end{array}$ & Redes Sociais \\
\hline
\end{tabular}


Avaliados os trabalhos sementes iniciou-se as buscas referentes ao Backward Snowballing. Foram analisadas, segundo os critérios de inclusão e exclusão, 98 trabalhos. Sendo selecionado 1, para fazer parte do conjunto de trabalhos relevantes à análise realizada neste MSL. Na busca referente ao Forward Snowballing foram analisadas 20 publicações, nenhuma delas foi selecionada para compor o conjunto final de trabalhos relevantes. As publicações que foram excluídas, assim foram por não atenderem aos critérios de inclusão ou exclusão estabelecidos no planejamento. Para melhor análise, a Tabela 4 dispõe de todos os trabalhos selecionados, de forma a sintetizar alguns dados da MSL. Na coluna 1 estão os títulos das publicações. A coluna 2 refere-se ao conjunto de trabalhos que a publicação pertence. Já a coluna 3 é composta pelo domínio de aplicação das soluções. A Condução do Mapeamento é finalizada ao se agrupar as publicações avaliadas a partir das buscas (Busca Scopus + Backward Snowballing + Forward Snowballing). A próxima seção responderá as QP a partir dos trabalhos selecionados.

\section{Relatório do MSL}

A primeira QP inicia a análise dos estudos selecionados a partir dos domínios abordados, de maneira a verificar se esses domínios estão diretamente relacionados à colaboração. (QP1) Quais são os domínios de aplicação tratados entre as soluções que adotam Interoperabilidade Social? Referentes ao domínio de Redes Sociais, Angarita et al. (2019) e Angarita et al. (2017) abordam o desenvolvimento de soluções para apoiar a interoperabilidade entre diferentes serviços de redes sociais online, de forma a promover a interoperabilidade social entre esses serviços. O domínio de Redes Sociais facilita o estudo da interoperabilidade social, devido às suas características e ao alto uso de redes sociais online, sendo uma área em constante expansão. Pessoas físicas e jurídicas interagem por meio das mídias sociais, o que contribui para a diversidade dos dados, e consequentemente uma maior possibilidade de contextualização social dos dados.

Ao investigarem aspectos de Cidades Inteligentes, Arbizzani et al. (2015) apresentam uma solução que utiliza objetos inteligentes para monitoramento das condições de construção e manutenção de edifícios. Nessa publicação, o termo interoperabilidade social é relacionado ao compartilhamento de informações para apoiar uma causa social. Monitores são instalados nas residências para possibilitar que os moradores, que apresentam baixo rendimento, tenham acesso ao consumo de energia elétrica em sua moradia. Essas informações permitem aos usuários controlar o consumo de energia elétrica, assim como, saber quais equipamentos elétricos consomem mais.

A solução apresentada por Vieira et al. (2016) busca, por meio de um modelo imagético personalizado de conhecimento, criar um catálogo de um acervo para melhorar as interoperabilidades cultural e social, de maneira a abordar a interoperabilidade social no domínio e Acervos Digitais. Hsu (2013) aborda a interoperabilidade social por meio de um sistema de recomendação de tags sociais. A solução propõe uma abordagem que utiliza do perfil de recurso semântico baseado em tags e na preferência do usuário para fornecer recomendações personalizadas de tags sociais.

A QP2 (Como as soluções abordam a Interoperabilidade Social?) explicita as soluções dos 5 trabalhos selecionados. Entre esses trabalhos não existem definições claras acerca de interoperabilidade social, apenas abordagens a partir de soluções implementadas. Contudo, essas abordagens permitem caracterizar a interoperabilidade social como um tipo de interoperabilidade que necessita da contextualização social e de um objetivo social para a troca dos dados. Essa caracterização contribui para a definição 
de requisitos para soluções de interoperabilidade social.

Hsu (2013) trata a recomendação de tags sociais em folksonomias. A solução consiste de um esquema que monitora a frequência de termos convencionais e ontologias para gerar, de forma automatizada, os perfis de recursos semânticos baseados nas tags. As ontologias são utilizadas pois as tags sociais, apesar de fornecerem os metadados e anotar os recursos da web, não dispõem de informações semânticas sobre elas e seus relacionamentos. Já as ontologias, apresentam esse aspecto semântico e oferecem suporte à representação do conhecimento, de maneira a contribuir para recomendação personalizada das tags socias. Assim, são apresentados modelos referentes aos usuários, perfis de usuários, as ontologias e tags sociais, além de um algoritmo. Esse algoritmo, gera de forma automatizada a lista de recomendação de tags sociais personalizadas. Portanto, Hsu (2013) aborda a interoperabilidade social de forma a considerar as informações de cunho social de cada uma das marcações para automatizar e promover a personalização e a recomendação das tags sociais.

Arbizzani et al. (2015) apresentam uma solução que utiliza de objetos inteligentes para monitoramento das condições de construção e manutenção de edifícios em uma região da Espanha. Para tanto, a interoperabilidade social ocorre quando as informações são compartilhadas com os responsáveis pelas edificações e os usuários. São adotados dispositivos inteligentes de monitoramento de energia. Por meio de um monitor os moradores são informados do consumo residencial. Os monitores foram instalados e tiveram um duplo papel no processo. Um deles foi a conscientização das famílias a fim de reduzir o consumo por meio de mudanças comportamentais. E o outro, foi o monitoramento das economias alcançadas devido a reforma da rede elétrica dos edifícios. A partir da análise dos consumos das residências foram sugeridas práticas para a diminuição desse consumo, de forma a conscientizar os moradores.

Vieira et. al (2016) propõem uma solução para apoiar a interoperabilidade social que consiste na utilização de imagens chaves para criação de catálogos de uma biblioteca digital. Uma imagem apresenta potencial de comunicação em massa devido a sua universalidade. Diante disso, os autores desenvolveram o conceito de um Catálogo Público Online (iOPAC) como um Knowledge Organization Systems (KOS) implementado de forma semelhante a um mecanismo focado no usuário compatível com o FRSAD (Requisitos Funcionais para Dados da Autoridade do Assunto). O FRSAD idealiza que um trabalho é composto de um assunto (tema), e um tema é descrito por um ou mais nomes. O iOPAC é atrativamente visual, o que aumenta a compreensão do Catálogo. Além de apresentar um conceito de portabilidade maior dos descritores, melhor interpretação e interoperabilidade entre os códigos e competências. Essas características potencializam a Interoperabilidade Social.

Diferente dos trabalhos anteriores, Angarita et al. (2019) e Angarita et al. (2017) focam na interoperabilidade entre Redes Sociais Online (OSNS) e serviços de comunicação. As soluções de interoperabilidade social adotadas propõem a interoperabilidade com o objetivo de facilitar a usabilidade e manutenibilidade dos diferentes perfis do mesmo indivíduo. E ainda, promover a interação e colaboração entre usuários que não estejam, necessariamente, utilizando do mesmo serviço. Angarita et al. (2017) focam na interoperabilidade entre serviços de comunicação independentemente do tipo de interação social desses serviços. Sendo assim, é proposto um Barramento de Comunicação Social que é executado em conjunto com o XSB (eXtensible Service Bus) e que apresentam paradigma arquitetônico semelhante. O XSB foi desenvolvido como 
um protocolo de barramento que promove a interoperabilidade entre serviços de interações heterogêneas no nível computacional. Já o Barramento de Comunicação Social trata da interação a nível humano. Angarita et al. (2019) se baseiam no trabalho anterior [Angarita et al. 2017] e desenvolvem o USNB (Universal Social Network Bus). O USNB é baseado no paradigma do barramento de serviços e promove a interação por meio de perfis sintéticos para acesso às diferentes OSNS.

Como visto nas QP exploradas, nenhuma das soluções são aplicadas diretamente para apoiar o desenvolvimento de um sistema colaborativo. Contudo, as soluções selecionadas abrangem os fatores sociais e abordam algumas soluções que podem promover a colaboração entre diferentes entidades que interoperam entre si. Assim, essa análise é feita por meio da QP3 (Como as soluções de Interoperabilidade Social encontradas podem apoiar a complexidade existente no desenvolvimento de sistemas colaborativos?). Hsu (2013) pode ser adaptado ao contexto de sistemas colaborativos por meio da utilização de tags sociais para apoiar a coordenação na formação de grupos de maneira automatizada. Metadados sociais dos colaboradores podem ser, em conjunto com dados técnicos, utilizados para apoiar a escolha das equipes de trabalho de acordo com a afinidade dos colaboradores por meio de marcações, por exemplo. Sendo assim, as tags sociais seriam ferramentas para marcação de elementos contextuais sociais em relação a cada colaborador. Seria criado um algoritmo que recomendasse colaboradores para uma mesma equipe de acordo com o significado dos dados interoperados entre os colaboradores, e com os objetivos de cada usuário.

No trabalho descrito por Arbizzani et al. (2015), a interoperabilidade social fica no campo da troca e gerenciamento de informação com um objetivo social. Os autores tratam do monitoramento de dados acerca da energia elétrica de um conjunto habitacional a fim de possibilitar a tomada de decisões e economia nas taxas de energia. É proposto um processo que apoia a cooperação, em busca de uma eficiência energética de uma região e possíveis novas soluções que permitam alcançar a eficiência e eficácia no consumo de energia elétrica. Elementos do projeto podem ser utilizados no desenvolvimento de sistemas colaborativos. O monitoramento do contexto social por meio de dispositivos inteligentes pode apoiar na interoperabilidade de dados relacionados aos colaboradores presentes em diferentes ambientes. O monitoramento de condições de saúde do colaborador, assim como do ambiente em que ele vive, pode proporcionar uma maior riqueza nos dados desse colaborador e apoiar na tomada de decisão dos coordenadores e outros colaboradores envolvidos. Em um processo de gerenciamento de tarefas, por exemplo, informações relacionadas à saúde podem definir a quantidade de tarefas e prazos de um colaborador. Colaboradores com uma saúde mais frágil podem demorar mais tempo para a entrega de tarefas definidas, acarretando um atraso no projeto. Para tanto, poderiam ser definidos um número menor de tarefas para esse colaborador e um apoio à saúde do mesmo. Os dados utilizados para essa contextualização podem ser captados por meio de dispositivos inteligentes vestíveis e interoperáveis.

Vieira et al. (2016) utilizam um modelo imagético para apoiar a interoperabilidade de informações em uma biblioteca digital. A utilização de "imagens-chave" na interface pode promover uma melhor interoperabilidade entre os códigos e as competências indexadas, promovendo a interoperabilidade social. A utilização de imagens para apoiar a representação das informações pode ser relevante para sistemas colaborativos. No gerenciamento do conhecimento dos processos pode-se interoperar imagens que abordem o contexto social dos colaboradores envolvidos. As imagens são munidas de um potencial 
cultural e social, pois são reconhecidas em diferentes regiões com o mesmo significado. Sendo assim, em sistemas colaborativos a indexação de imagens pode apoiar a percepção dos colaboradores envolvidos e fazer com que o sistema troque dados a partir das imagens reconhecidas e escolhidas por esses colaboradores, de maneira a considerar a representação que a imagem tem naquele meio social.

Angarita et al. (2019), (2017) abordam a interoperabilidade social a partir de diferentes redes sociais. O potencial colaborativo da interoperabilidade social é abordado entre o sistema Slack e outras redes sociais. Entretanto, uma limitação no que se refere à criação de perfis no Slack foi encontrada. Com isso, para interoperar com o Slack é criado um perfil USNB, denominado SocialBus. Esse perfil possibilita a interoperabilidade social a partir do alcance de usuários de outras redes sociais de maneira indireta. A solução desenvolvida ainda aborda seu potencial colaborativo a partir da argumentação de que o USNB contribui para uma maior inclusão em sistemas participativos.

\section{Interoperabilidade Social e Colaboração}

Os trabalhos previamente encontrados têm em comum a ideia de que a interoperabilidade social está relacionada à contextualização social dos dados, às relações e interações dos envolvidos nos processos de interoperabilidade e ao objetivo da interoperabilidade. Foram observadas também que a interoperabilidade social engloba elementos culturais, regras e políticas, que são fatores influenciadores do contexto do usuário e do ambiente em que se encontra. Eles são capazes de ditar os objetivos e comportamentos de um usuário ou sistema. Além disso, a interoperabilidade social está relacionada a outros níveis discutidos na literatura, que são as interoperabilidades sintática, semântica e pragmática. Pode-se analisar que a interoperabilidade social ocorre concomitante com pelo menos um desses níveis, focando no contexto e objetivos sociais.

No contexto de sistemas colaborativos, o caráter social e humano desse tipo de interoperabilidade possibilita a contextualização dos dados para que os colaboradores possam encontrar pontos em comum, de maneira a melhorar os relacionamentos para a condução das atividades. $\mathrm{O}$ entendimento dos fatores socioculturais em uma equipe distribuída geograficamente é valioso. Por exemplo, diferentes países podem apresentar cargas horárias de trabalho diferentes. Dessa forma, a partir do contexto social, e de forma automática, o sistema pode definir as atividades a serem realizadas pelo colaborador de acordo com as diferentes normas e regras contextualizadas. Além disso, a partir da contextualização social dos dados, o sistema pode promover a percepção do colaborador frente às mensagens trocadas, de maneira a aumentar o conhecimento relacionado às tarefas e à equipe.

A exemplo, propõe-se um cenário com uma equipe de desenvolvimento de software geograficamente distribuída. O desenvolvimento distribuído de software envolve várias aplicações e ferramentas que necessitam interoperar para a construção do produto final. Para apoiar a colaboração dessa equipe propõe-se a captura e o gerenciamento de dados de cunho social dos desenvolvedores. A captura e o gerenciamento desses dados podem ser usados para a observação das condições que influenciam o trabalho de cada um dos membros da equipe. Como resultado, contribuem para a tomada de decisão relacionada às condições de trabalho dos membros, recursos motivacionais que podem ser aplicados, investimentos a serem realizados e alterações no quadro de desenvolvedores. Esses dados são importantes também para avaliar as interações entre os colaboradores. 


\section{Conclusão}

Este trabalho propôs analisar aspectos de interoperabilidade social para apoiar a construção de sistemas colaborativos. A Interoperabilidade Social ainda é um tipo de interoperabilidade pouco explorado na literatura. Para tanto, foi conduzido um MSL para que fosse investigado o estado da arte das pesquisas acerca da interoperabilidade social e da sua relação com os conceitos de colaboração. Para a condução deste MSL foi utilizada a Busca Híbrida. Essa metodologia permite a elaboração e reprodução do processo de mapeamento com redução da possibilidade de viés e da perda de trabalhos relevantes.

Como contribuição, este trabalho explora a Interoperabilidade Social, assim como a relação que este nível de interoperabilidade tem com outros níveis e com a troca de dados em sistemas colaborativos. Percebemos que ainda existem questões abertas para a formulação de novas soluções de interoperabilidade para apoiar as diversas formas de colaboração. Como trabalhos futuros, pretende-se explorar aspectos relacionados à interoperabilidade social, assim como a sua relação com outros níveis de interoperabilidade no contexto de sistemas colaborativos. Por exemplo, desenvolver soluções que possam utilizar da contextualização social para apoiar os processos de desenvolvimento de sistemas colaborativos e atender os objetivos sociais demandados nesses processos.

\section{References}

Angarita, R., Lefèvre, B., Ahvar, S., Ahvar, E., Georgantas, N., \& Issarny, V. (2019) "Universal Social Network Bus: Toward the Federation of Heterogeneous Online Social Network Services", ACM Transactions on Internet Technology (TOIT), 19(3), p. 1-19.

Angarita, R., Georgantas, N., Parra, C., Holston, J., \& Issarny, V. (2017) "Leveraging the service bus paradigm for computer-mediated social communication interoperability", In: 2017 IEEE/ACM 39th International Conference on Software Engineering: Software Engineering in Society Track (ICSE-SEIS), p. 67-75.

Arbizzani, E., Civiero, P., Madrigal, L. O., \& Lanzarote, B. S. (2015) "Smart solutions for low-income buildings rehabilitation: international researches and experiences", TECHNE-Journal of Technology for Architecture and Environment, p. 222-231.

Chen, W. \& Quan-Haase, A. (2020) "Big data ethics and politics: Toward new understandings", Social Science Computer Review, 38(1), p. 3-9.

David, J. M. N., \& Maciel, R. S. P. (2009) "WGWSOA-implementing collaboration services in a middleware infrastructure”, In: 2009 Simpósio Brasileiro de Sistemas Colaborativos, IEEE, p. 193-201.

Garlapati, R. and Biswas, R. (2012) "Interoperability in Healthcare: A focus on the Social Interoperability", (Dissertation), Retrieved from http://urn.kb.se/resolve?urn=urn:nbn:se:bth-5468

Hsu, I. C. (2013) "Integrating ontology technology with folksonomies for personalized social tag recommendation", Applied Soft Computing, 13(8), p. 3745-3750.

Kasunic, M. (2001) "Measuring systems interoperability: Challenges and opportunities", Carnegie-Mellon Univ Pittsburgh Pa Software Engineering Inst., p. 3-8. 
Landsbergen Jr, D. and Wolken Jr, G. (2001) "Realizing the promise: Government information systems and the fourth generation of information technology", Public administration review, 61(2), p. 206-220.

Lesh, K., Weininger, S., Goldman, J. M., Wilson, B., and Himes, G. (2007), "Medical device interoperability-assessing the environment", In: 2007 Joint Workshop on High Confidence Medical Devices, Software, and Systems and Medical Device Plug-andPlay Interoperability (HCMDSS-MDPnP 2007), IEEE, p. 3-12.

Li, V., Liu, K., \& Liu, S. (2013), "Semiotic interoperability-a critical step towards systems integration", In: International Conference on Knowledge Discovery and Information Retrieval and the International Conference on Knowledge, Management and Information Sharing, p. 508-513.

Maciel, R. S. P., David, J. M. N., Claro, D., \& Braga, R. (2017). Full interoperability: Challenges and opportunities for future information systems. Sociedade Brasileira de Computação, p. 107-116.

Mourão, E., Kalinowski, M., Murta, L., Mendes, E., \& Wohlin, C. (2017) "Investigating the use of a hybrid search strategy for systematic reviews", In: 2017 ACM/IEEE International Symposium on Empirical Software Engineering and Measurement (ESEM), IEEE, p. 193-198.

Neiva, F. W., David, J. M. N., Braga, R., and Campos, F. (2016) "Towards pragmatic interoperability to support collaboration: A systematic review and mapping of the literature", Information and Software Technology, 72, p. 137-150.

Neiva, F. W., David, J. M. N., Braga, R., Campos, F., and Freitas, V. (2015) "Prime: Pragmatic interoperability architecture to support collaborative development of scientific workflows", In: 2015 IX Brazilian Symposium on Components, Architectures and Reuse Software, IEEE, p. 50-59.

Olshannikova, E., Olsson, T., Huhtamäki, J., \& Kärkkäinen, H. (2017) “Conceptualizing big social data", Journal of Big Data, 4(1), 3.

Petticrew, M., \& Roberts, H. (2008) "Systematic reviews in the social sciences: A practical guide", John Wiley \& Sons.

Ruban, K., Gavrilova, I., and Novikova, T. (2019) "Development of interoperability problem-oriented model for university e-learning system (by the case of nosov magnitogorsk state technical university)", In: 2019 International Multi-Conference on Industrial Engineering and Modern Technologies (FarEastCon), IEEE, p. 1-5.

Sadeghi, P., Benyoucef, M., and Kuziemsky, C. E. (2012) “A mashup-based framework for multilevel healthcare interoperability", Information systems frontiers, 14(1), p. 5772.

Soares, D. D. S. (2010) "Interoperabilidade entre sistemas de informação na Administração Pública”, (Tese de Doutorado), In: Universidade do Minho, http://hdl.handle.net/1822/10539.

Vieira, S. B., De Brito, M., El Hadi, W. M., \& Zumer, M. (2016) "Developing Imaged KOS with the FRSAD Model: A Conceptual Methodology", In: NKOS@ TPDL, p. $36-47$. 